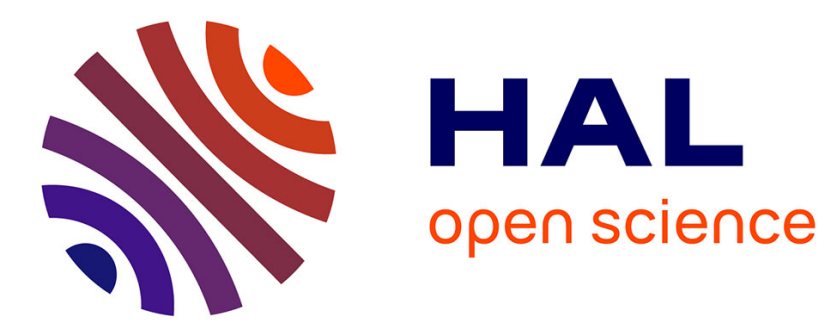

\title{
Performance evaluation of CUDA programming for 5 -axis machining multi-scale simulation
}

Felix Abecassis, Sylvain Lavernhe, Christophe Tournier, Pierre-Alain Boucard

\section{To cite this version:}

Felix Abecassis, Sylvain Lavernhe, Christophe Tournier, Pierre-Alain Boucard. Performance evaluation of CUDA programming for 5-axis machining multi-scale simulation. Computers in Industry, 2015, 71, pp.1-9. 10.1016/j.compind.2015.02.007 . hal-01136770

\author{
HAL Id: hal-01136770 \\ https://hal.science/hal-01136770
}

Submitted on 1 Apr 2015

HAL is a multi-disciplinary open access archive for the deposit and dissemination of scientific research documents, whether they are published or not. The documents may come from teaching and research institutions in France or abroad, or from public or private research centers.
L'archive ouverte pluridisciplinaire HAL, est destinée au dépôt et à la diffusion de documents scientifiques de niveau recherche, publiés ou non, émanant des établissements d'enseignement et de recherche français ou étrangers, des laboratoires publics ou privés. 


\title{
Performance Evaluation of CUDA programming for 5-Axis machining multi-scale simulation
}

\author{
Felix Abecassis ${ }^{\mathrm{a}}$, Sylvain Lavernhe ${ }^{\mathrm{a}}$, Christophe Tournier ${ }^{\mathrm{a}, *}$, Pierre-Alain \\ Boucard $^{\mathrm{b}}$ \\ ${ }^{a}$ LURPA, ENS Cachan, Univ Paris-Sud \\ 61 Avenue du président Wilson, F-94235 Cachan, France \\ ${ }^{b}$ LMT Cachan, ENS Cachan/CNRS/PRES UniverSud Paris \\ 61 Avenue du président Wilson, F-94235 Cachan, France
}

\begin{abstract}
5-Axis milling simulations in CAM software are mainly used to detect collisions between the tool and the part. They are very limited in terms of surface topography investigations to validate machining strategies as well as machining parameters such as chordal deviation, scallop height and tool feed. Z-buffer or $\mathrm{N}$-Buffer machining simulations provide more precise simulations but require long computation time, especially when using realistic cutting tools models including cutting edges geometry. Thus, the aim of this paper is to evaluate Nvidia CUDA architecture to speed-up Z-buffer or N-buffer machining simulations. Several strategies for parallel computing are investigated and compared to single-threaded and multi-threaded CPU, relatively to the complexity of the simulation. Simulations are conducted with two different configurations including Nvidia Quadro 4000 and Geforce GTX 560 graphic cards.

Keywords: 5-Axis milling, Machining simulation, GPU computing, GPGPU, CUDA architecture
\end{abstract}

\section{Introduction}

All manufactured goods present surfaces that interact with external elements. These surfaces are designed to provide sealing or friction functions, op-

\footnotetext{
*Corresponding author Tel.: +331474027 52; Fax: +331474022 20

Email address: christophe.tournier@ens-cachan.fr (Christophe Tournier)
} 
tical or aerodynamics properties. The technical function of the surface requires specific geometrical deviations which can be performed with specific manufacturing process. In particular, to reduce the cycle time of product development, it is essential to simulate the evolution of geometrical deviations of surfaces throughout the manufacturing process. Currently, simulations of the machined surfaces in CAM software are very limited. These simulations are purely geometric where cutting tools are modeled as spheres or cylinders without any representation of cutting edges.

In addition, these simulations do not include any characteristic of the actual process that may damage the surface quality during machining such as feedrate fluctuations, vibrations and marks caused by cutting forces and tool flexion, geometrical deviations induced by thermal expansion, etc. Many models exist in the literature [1][2][3], but given their complexity and specificity, they often require very high computation capability and remains academic models. Finally, CAM software simulations do not provide the required accuracy within a reasonable computation time and the selection of an area in which the user wishes to have more precision is impossible.

However, there are many methods to perform machining simulations in the literature: methods based on a partition of the space by lines [4], by voxels [5] or by planes [6], methods based on meshes [7], etc. If we consider the Z-buffer or N-buffer methods applied to a realistic description of both the tools and the machining path, earlier work have shown that it is possible to simulate the resulting geometry on small portions of the surface in a few minutes [8][9]. Simulation results are very close to experimental results but the simulated surfaces have an area of a few square millimeters with micrometer resolution (Fig. 1). Therefore, the limits in terms of computing capacity and simulation methods restrict the realistic simulations of geometrical deviations.

A faster simulation could help to take into account more complex and realistic geometrical model for the tool and its displacements and rotations onto the workpiece and introduce non purely geometrical model but mechanical behavior with cutting loads, tool and workpiece flexions. Polishing process could be 
investigated considering abrasive particles as elementary cutting tools, increasing drastically the number of intersection tests without increasing the time for simulation.

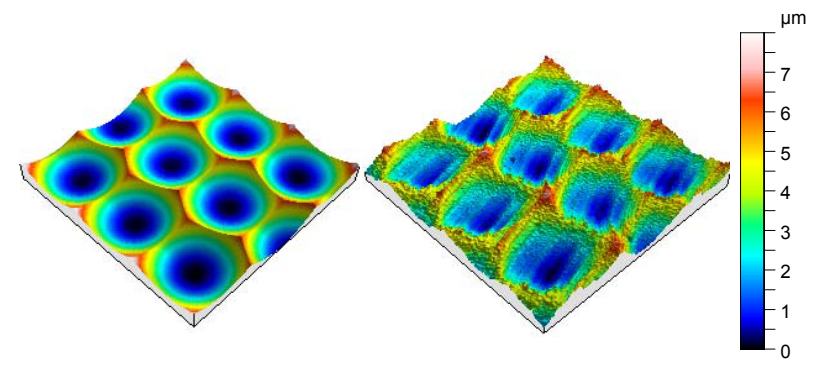

Figure 1: Simulation versus experimental results for 5-Axis milling with ball-end tool

Regarding the hardware, NVIDIA has recently developed CUDA, a parallel processing architecture for faster computing performance, harnessing the power of GPUs (graphics processing units) by using GPGPU. GPGPU (generalpurpose computing on graphics processing units) is the utilization of a GPU, which typically handles computation only for computer graphics, to perform computation in applications traditionally handled by the central processing unit (CPU). GPU's technology in the CAD/CAM community is widely used but the CUDA technology is just beginning in the field of machining.

Few works can be underlined such as those proposed in [10] dealing with the acceleration of a specific tool path algorithm called virtual digitizing. It is based on a Z-map approach and similar to the inverse Z-buffer developed in [11] [12] which is suited to parallel processing. They study in particular the influence of the calculations in single or double precision on performances on both GeForce or Quadro devices. Kaneko et al. have also used the CUDA technology to speed-up the cutter workpiece engagement in 5-Axis machining [13]. They did not investigate the traditional approach based on Voxelmap or Z-buffer but a novel approach based on swept volume, discretization of the tool flutes and a polygonal approximation of the workpiece. Inui et al. compared two GPU based acceleration methods for the geometric 3-Axis milling simulation: one based on 
the depth buffer and the other based on the CUDA architecture [14]. Regardless of the computation speed with CUDA, limits can be reached because of costly data exchanges between CPU and GPU memories. Hence speed-up seems to be efficient for small toolpath regardless of the tool size.

The aim of this paper is thus to propose a calculation scheme harnessing the CUDA parallel architecture to reduce the computation time despite the very large number of elementary computations. Within the multi-scale simulation context, this consists in proposing the best distribution of the calculation according to the configuration of the case studies and especially the zoom level.

After having presented constraints related to the use of the CUDA architecture, the machining simulation algorithm to be parallelized is recalled. Then, different solutions to the problem of parallelization with CUDA are investigated. Finally a comparison of the computing time between GPU and CPU processors is proposed on several 3- and 5-Axis milling examples.

\section{Compute unified device architecture}

CUDA is a parallel computing platform that unlocks programmability of NVIDIA graphic cards in the goal of speeding up the execution of algorithms exhibiting a high parallelization potential. Algorithms are written using a modified version of the ANSI C programming language (CUDA C) and can consequently be executed seamlessly on any CUDA capable GPU [15].

The strength of the CUDA programming model lies in its capability of achieving high performance through its massively parallel architecture (Fig. ??). In order to achieve high throughput, the algorithm must be divided into a set of tasks with minimal dependencies. Tasks are mapped into lightweight threads which are scheduled and executed concurrently on the GPU. Thirty-two threads are grouped to form a warp. Threads within the same warp are always executed simultaneously, maximum performance is therefore achieved if all the 32 threads are executing the same instruction at each cycle. Warps are them- 
selves grouped into virtual entities called blocks, the set of all blocks forms the grid, representing the parallelization of the algorithm. Threads from the same block can be synchronized and are able to communicate efficiently using a fast on-chip memory, called shared memory, whereas threads from different blocks are executed independently and can only communicate through global (GDDR) memory of the GPU.

The number of threads executing simultaneously can be two orders of magnitude larger than on a classical CPU architecture. As a consequence, task decomposition should be fine-grained opposed to the traditional coarse-grained approach for CPU parallelization. The combination of this execution model and memory hierarchy advocates a multi-level parallelization of the algorithm with homogeneous fine-grained tasks, dependent tasks should be mapped to the same warp or block in order to avoid costly accesses to global memory. In order to harness the power of the massively parallel architecture of GPUs, a complete overhaul of the algorithm is often required [16].

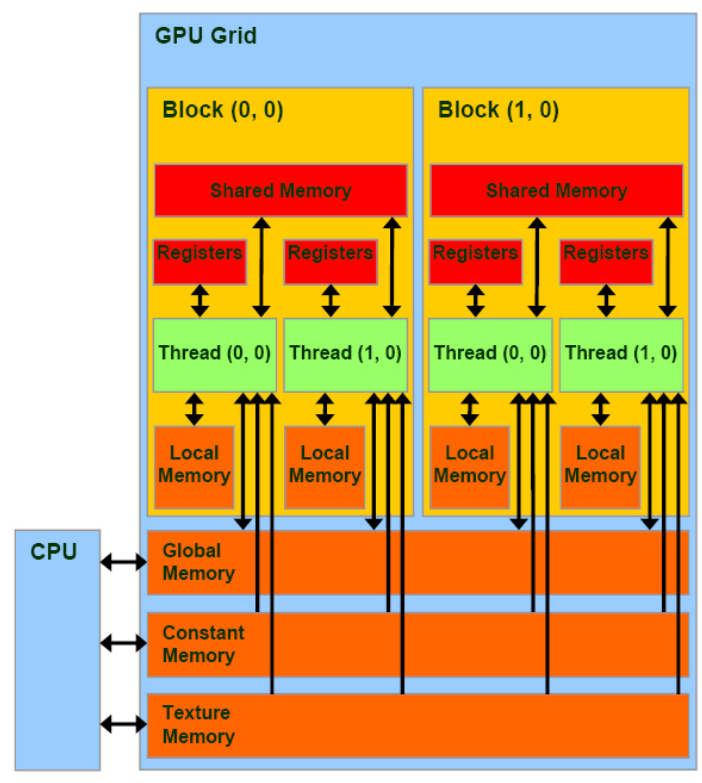

Figure 2: Cuda architecture 
The scalability of the CUDA programming models stems from the fact that, thanks to this fine-grain task model, the number of threads to execute generally exceeds the number of execution units on the GPU, called CUDA cores, for those threads. As more CUDA cores are added, e.g. through hardware upgrades, performance will increase without requiring changes to the code. Another benefit of this fine-grained decomposition is the ability to hide latency, if a warp is waiting for a memory access to complete, the scheduler can switch to another warp by selecting it from a pool of ready warps. This technique is called thread level parallelism (TLP). Latency hiding can also be done within a thread

directly, by firing several memory transactions concurrently, this technique is called instruction level parallelism (ILP).

Despite continuous efforts by NVIDIA to improve the accessibility of CUDA, the learning curve remains steep. Attaining high performance requires careful tuning of the algorithm through a detailed knowledge of the CUDA execution model.

\section{Computation algorithm}

The computation algorithm relies on the Z-buffer method [4]. This method consists in partitioning the space around the surface to be machined in a set of lines which are equally distributed in the $\mathrm{x}-\mathrm{y}$ plane and oriented along the z-axis. The machining simulation is carried out by computing the intersections between the lines and the tool along the tool path. The geometry of the tool is modeled by a sphere or a triangular mesh including cutting edges which allows to simulate the effect of the rotation of the tool on surface topography.

The tool path is whether a 3 -axis tool path with a fixed tool axis orientation or a 5-Axis tool path with variable tool axis orientations. In order to simulate the material removal, intersections with a given line are compared and the lowest is registered (Fig. 3).

The complete simulation requires the computation of the intersections between the $N$ lines and the $T$ triangles of the tool mesh at each tool posture $P$ 
on the tool path. The complexity $C$ of the algorithm is thus defined by :

$$
C=N \times T \times P
$$
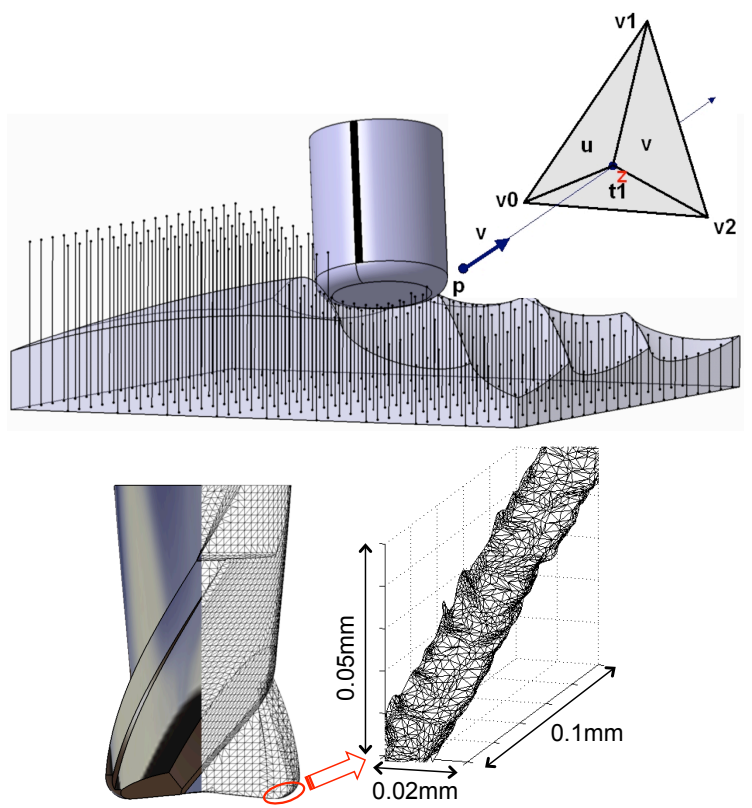

Figure 3: Z-buffer simulation method and cutting edge

If we consider a finish operation on a $5 \mathrm{~mm} \times 5 \mathrm{~mm}$ patch of surface with a resolution of $3 \mu \mathrm{m}$, this leads to 2,250,000 lines. The tool mesh contains 3000 triangles and the tool path contains 70,000 postures including the tool rotation around the spindle axis with a discretization step of 0.2 degrees. The number of intersections to compute is equal to $4.7 \cdot 10^{14}$. This technique can be accelerated by decreasing the number of tests by first calculating the intersection with the bounding box of the tool and using data structures such as bounding volume hierarchy $[17]$ or binary space partitioning trees [18].

In the case of Z-buffer, all lines are oriented in the same direction which allows many optimizations on both the number of intersection tests to be performed and the number of operations required for each test. For each triangle, its projection on the grid and its 2D bounding box is calculated (Fig. 4). The 
lines outside the bounding box (shaded circles) are not tested, they can not have an intersection with the triangle. A double loop is then performed to test the intersection of each line within the bounding box with the 2D projection of the triangle. The lines may intersect with the triangle (green) or not (red). If the intersection exists, the height is calculated and then compared with the current height of the line.

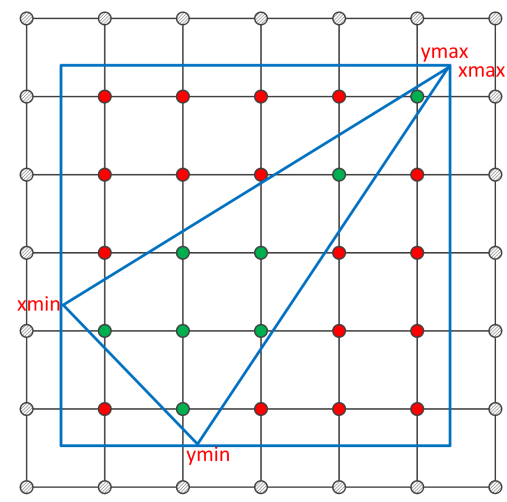

Figure 4: 2D bounding box

As there are no dependancies between the milling process at different locations on the tool path, each of these intersections could be carried out simultaneously. However, due to memory limitations and tasks scheduling, the parallel computing of these intersections on graphics processing units with CUDA has to be done carefully.

\section{Line triangle intersection issues}

The intersection algorithm is using the barycentric coordinates to check whether the intersection point with the line is inside the triangle or not. This requires to position the triangle relative to the line. For example, for a translation of the cutting tool, computing the reference frame ( $\mathrm{u}$ and $\mathrm{v}$ coordinates) formed by the two edges of the triangle can be done before or after the translation. Adding a large number (the coordinates of the translation) and a small 
number may cause a rounding error [19]. Thus, for a better numerical stability it is preferable to perform the calculation prior to translation.

Let us consider the triangle $t_{1}$ formed of three vertices: $a=(0,0,2), b=$ $(0,3.1,5.1)$ and $c=(3.1,0,7)$. Also consider the same triangle after a translation $(d, d, d)$ with $d=1024$ (value that can be represented exactly in single precision). We call this triangle $t_{2}$. Tables 1 shows the impact on the calculation steps when computing the intersection of $t_{1}$ with the lines $l_{1}=(1.4,1.4,0)$ and $l_{2}=$ $l_{1}+(d, d, d)$.

Table 1: Calculation steps

\begin{tabular}{|c|c|c|c|}
\hline & $\mathrm{u}$ & $\mathrm{v}$ & dist \\
\hline$t_{1} / l_{1}$ & $3.4 \mathbf{1 0 0 0 0 0 8}$ & 4.33999967 & $5.3580 \mathbf{6} 465$ \\
\hline$t_{2} / l_{2}$ & 3.40989756 & 4.34004163 & $d+5.35803223$ \\
\hline
\end{tabular}

As expected, the coordinates $u$ and $v$ and the intersection distance are different between the two cases, which can lead to wrong conclusion on whether the line intersects the triangle or not, but also on the calculated distance.

In addition, the coordinates $x$ and $y$ of the grid point underlying the line are very close to coordinates $x$ and $y$ of triangles, while the $z$ coordinates are very different. Adding and subtracting values with very different exponents also implies a loss of precision. To reduce this source of error, it is proposed to modify the algorithm and perform the intersection in the triangle coordinate system by applying the reverse translation to the line, except for the $z$ coordinate. In this way, there is no loss of precision on the triangle coordinates, since all coordinates of the vertices and lines are of the same order of magnitude. The initial algorithm is shown in Fig. 5, the improved version is then shown in Fig. 6.

We make the assumption that on the same architecture, result in double precision is necessarily more accurate (closer to the actual mathematical result) than result in single precision. The computation on the CPU Xeon 64-bit is then considered as the reference. The GPU 64-bit results being similar to CPU 


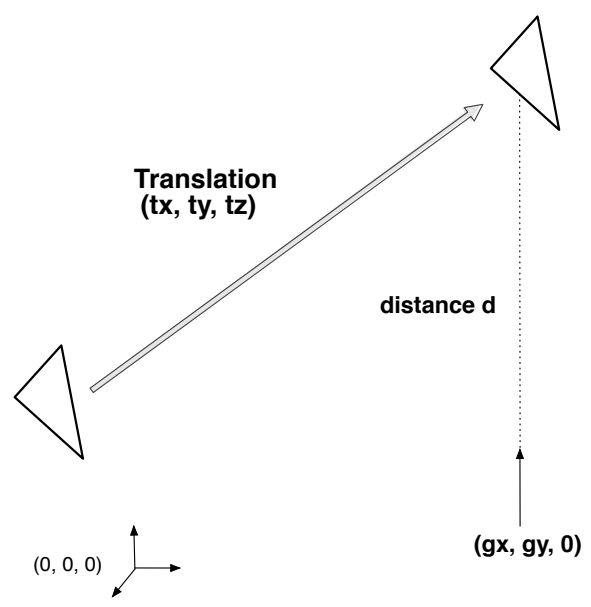

Figure 5: Initial algorithm

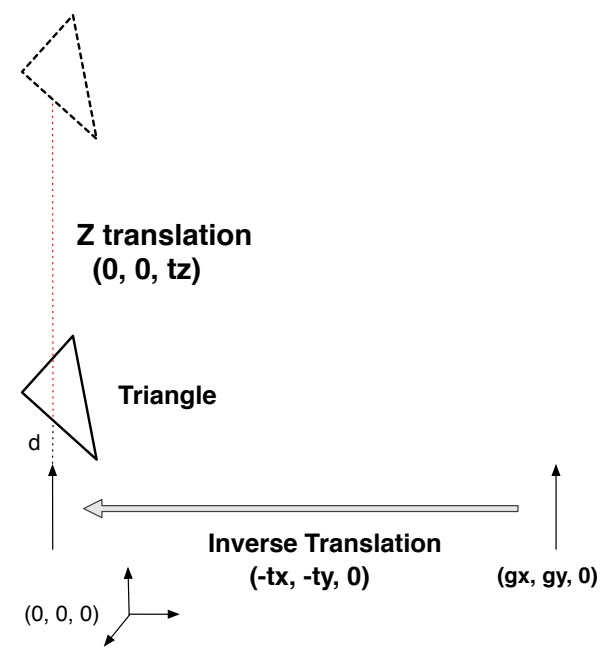

Figure 6: Improved algorithm 
in 64-bit, only the results on GPU 32-bit are presented. The case of study is spiral and the result on the GPU is shown Fig. 7. The error shown is absolute, for lines of initial height equal to 1000. Fig. 7 shows that in single precision, the improved algorithm generates numerical errors 100x smaller than the initial one. Consider for instance the machining simulation of a car bumper, given Z-buffer lines of $1000 \mathrm{~mm}$ height, the error due to single precision is $0.001 \mathrm{~mm}$ with the initial algorithm and $0.00001 \mathrm{~mm}$ with the improved one. Thus the improved algorithm is used in further computations.
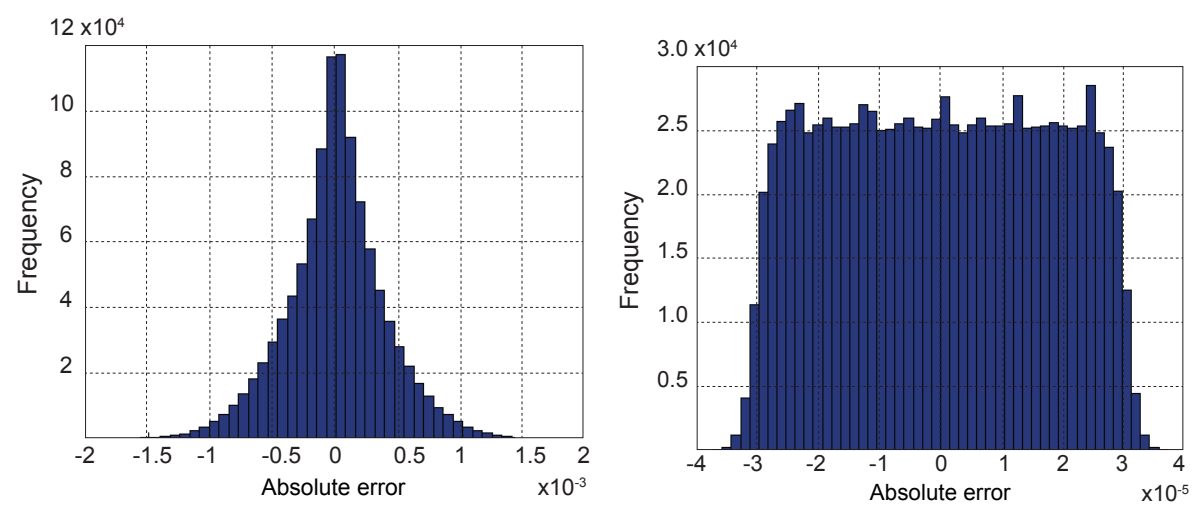

Figure 7: Single precision error for initial and improved algorithm

\section{Parallel computation strategies}

In the case of parallel execution, computation time is always limited by the longest run. A long running thread can slowdown an entire warp, or worse, an entire block. If the granularity of tasks is too high, this situation is more likely to occur. The CUDA scheduler present on each graphics card is optimized for the processing of a set of tasks having homogeneous complexities. In many cases, this choice proves to be very effective. However in cases where the execution time per task has a large variance, this method may not be optimal.

The basic algorithm consists in determining whether there is an intersection between a line and a triangle associated to a position of the tool. Given these 
three variables on which the algorithm iterates during the sequential computation, there are numerous possible combinations to affect threads and browse the set of lines, triangles and positions. These combinations may generate unfavorable cases in terms of parallelization and computational time. The most interesting possibilities are summarized in Table 2 and developed hereafter.

Table 2: Different strategies

\begin{tabular}{cc} 
Line: One thread $=$ One line \\
\hline For each position & For each triangle \\
For each triangle & For each position \\
Intersection & Intersection
\end{tabular}

Coarse: One thread $=$ One position

For each triangle

For each line

Intersection

Inverted: One thread $=$ One triangle

For each position

For each line

Intersection

Fine: One thread $=$ One triangle for one pos.

For each line

Intersection

\subsection{The line approach}

Each CUDA thread is assigned to a line of the Z-buffer grid. A thread calculates the intersection of the line with the tool triangles for all positions along the path. To reduce the number of intersection tests, the intersection with the global bounding box of the tool in a given position is first calculated. 
If there is intersection, each triangle of the tool is then tested because a finer bounding box cannot be used (Fig. 4). The advantage of this approach is that there is no need to use atomic operations since the minimum height of cut for each line is calculated by a single thread. However, for each position of the tool, the transformation matrix must be computed which significantly increase the number of calculations. Finally, this approach leads to longer computation time and is not further considered.

Then, we only consider strategies that allow reducing the amount of required calculations, i.e. those that reduce the calculation area by fine bounding box and that let the geometric transformation (to position the mesh relatively to the lines) outside the thread.

\subsection{The tool position approach (coarse)}

Each thread is assigned to a position of the tool and applies the Z-buffer algorithm for each triangle of the tool mesh for this position. The granularity of tasks is high: if the amount of triangles to be processed is large, each thread will run for a long time. If the computation time between threads is heterogeneous, some threads of a warp may no longer be active, and therefore the parallelism is lost. A thread may affect the cutting height of several lines so a line can be updated by multiple threads and global memory access conflicts appear. Atomic operations proposed by CUDA are then used to allow concurrent update of the height of the lines. This method is the one implemented in the CPU configuration.

\subsection{The tool triangle approach (inverted)}

Each thread is assigned to a triangle of the tool mesh and applies the Z-buffer algorithm for the triangle for all positions along the path. In the case of the 3 -axis machining, the dimensions of the triangle projection on the grid remain constant which allows optimization possibilities. However, in 5-Axis milling, this property is no longer valid. Each position involves a new transformation matrix to be recovered in memory which increases significantly the number of 
memory transactions. This method is advantageous in the case of a 3-axis tool path but is not suited for 5-Axis tool path. The same remarks apply the previous approach on the granularity of tasks and conflicts in global memory access.

\subsection{The triangle and position approach (fine)}

In this approach, each thread is assigned to a single triangle in one position. As the granularity is smaller, the risk of a bad balance workload disappears. However, in return the number of threads is much higher: the management and scheduling of billions of threads involve additional workload for the task scheduler CUDA and thus a significant increase in computation time.

\section{Numerical investigations}

\subsection{Case study}

Different configurations of trajectories and tools have been used for testing. A first setting, called random (Fig. 8(a)), for which random positions are generated on a plane and a second setting, where a plane is machined along a downward spiral called spiral (Fig. 8(b)). In both cases, the ball-end tool is 100 times smaller compared to the dimensions of the surface.

A roughing operation with a torus tool (Fig. 8(c)) and a 5-Axis finishing operation with a ball end tool (Fig. 8(d)) of a pseudo blade part are also proposed in the benchmark. At last, a small scale simulation benchmark, called Cutting edge, is proposed with the mesh of a ball end mill including worn cutting edges and spindle rotation (Fig. 8(e)) which lead to numerous tool positions and large tool mesh size (Table 3).

One of the objectives is to be able to dynamically zoom on the part and update the simulation. Thus the grid size is constant and set to $1024 \times 1024$ lines, regardless of the zoom factor. Therefore, the above configurations are also studied with or without a zoom on the surface, which changes the size of the problem (Tables 3 and 4). 
Table 3: Macro geometry (without zoom)

\begin{tabular}{lccc} 
Case & Tool position P & Tool & Mesh size T \\
\hline Random & 200000 & sphere & 320 \\
Spiral & 200000 & sphere & 1984 \\
Roughing & 47837 & torus & 25904 \\
Finishing & 53667 & sphere & 12482 \\
Cutting edge & 144001 & local edges & 36389 \\
\hline
\end{tabular}

Table 4: Micro geometry (with zoom)

\begin{tabular}{lccc} 
Case & Tool position $\mathrm{P}$ & Tool & Mesh size T \\
\hline Random & 135 & sphere & 320 \\
Spiral & 206 & sphere & 1984 \\
Roughing & 619 & torus & 25904 \\
\hline
\end{tabular}

Two different dardware configurations are considered:

- $\mathrm{CAD} / \mathrm{CAM}$ configuration:

- CPU1: Intel Xeon X5650 2.67 GHz, 6 cores, 12 virtual cores, SSE, 64 Gflops DP, (\$1000)

- GPU1: Nividia Quadro $40000.95 \mathrm{GHz}, 8$ multiprocessors, 256 CUDA Cores, 243 Gflops DP, 486 SP, (\$700)

- GAMING configuration:

- CPU2: AMD Phenom II X4 955 (3.2 GHz), 4 cores, SSE, 32 Gflops $\mathrm{DP},(\$ 150)$

- GPU2: Nividia GeForce GTX 5601.76 GHz, 8 multiprocessors, 384 CUDA Cores, 1264 Gflops SP, $(\$ 220)$

Thus, from a purely theoretical standpoint, the GPU Quadro 4000 is approximately 4x faster than the Xeon CPU in 64-bit and the GPU GTX 560 40x faster than the AMD Phenom. This will help us to discuss the results in section 6.3. 


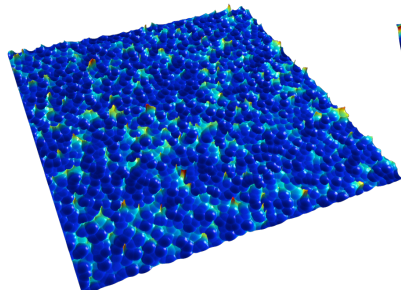

(a) random

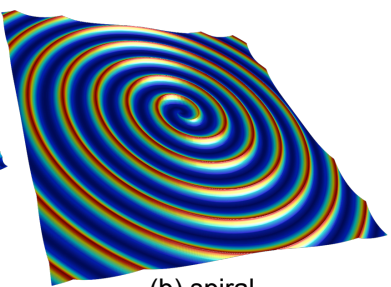

(b) spiral

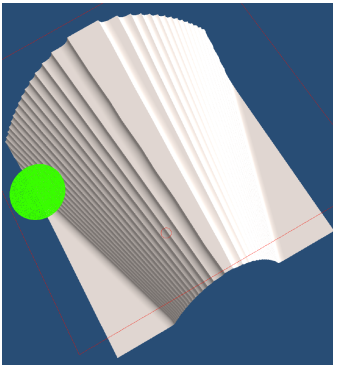

(c) roughing

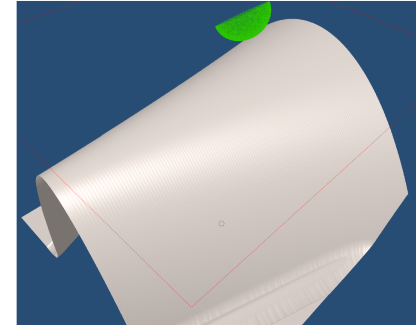

(d) finishing

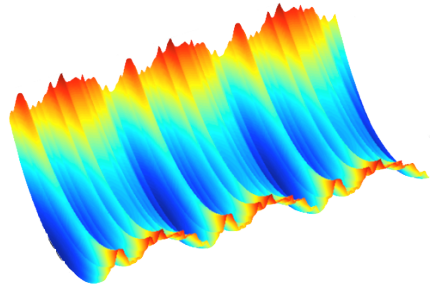

(e) cutting edge

Figure 8: Cases of study 


\subsection{Discussion on computation strategies}

Excluding cases of zoom, GPU implementation is on average 5 times faster than the CPU implementation with the engine GPU Coarse (1 thread handles all triangles in one position).

When the number of triangles in the mesh of the tool is too low, the number of threads used in the engine GPU Inverted is much lower than the number of threads that can be executed simultaneously on all multiprocessors. Performance is worse (random). When the number of triangles increases (random to spiral, spiral to roughing and roughing to cutting edge), performance improves accordingly. Furthermore, as mentioned earlier, acceleration with bounding box can't be done in 5-Axis machining (finishing) which leads to smaller speed-up compared to 3 -axis operations.

With the engine GPU Fine (1 thread processes one triangle in one position), there are too few lines in the bounding box of each triangle because the triangles are very small compared to the size of the grid. Each thread is little busy and time is lost to launch these threads.

\begin{tabular}{|c|c|c|c|c|}
\hline \multirow[t]{2}{*}{ Case } & \multicolumn{4}{|c|}{ GPU Speed-up } \\
\hline & $\mathrm{CPU}$ & Coarse & Inverted & Fine \\
\hline Random & 366 & 5.3 & 0.4 & 3 \\
\hline Spiral & 1061 & 4.3 & 2.9 & 2.1 \\
\hline roughing & 7891 & 5.1 & 5.1 & 1.7 \\
\hline Finishing & 11542 & 4.9 & 3.6 & 4.2 \\
\hline Cutting edge & 22882 & 8.3 & 7.8 & 2.3 \\
\hline
\end{tabular}

In micro geometry configurations, the GPU speed-up does not meet expectations (Table 4). Since the number of positions with zoom is much lower than the number of threads that can be executed simultaneously on all GPU multiprocessors, the granularity of the simulation engine GPU Coarse is too high: the available parallelism is not exploited. The computation engine must exhibit a much finer granularity, such as the GPU Fine engine, that should be used 
to make best use of the number of threads.

Table 6: Micro geometry $\mathrm{N}=1024 * 1024 ; 64$ bits ; CAD/CAM configuration
\begin{tabular}{lcccc} 
Case & Time $(\mathrm{ms})$ & \multicolumn{3}{c}{ GPU Speed-up } \\
& CPU & Coarse & Inverted & Fine \\
\hline Random & 168 & 0.14 & 0.22 & 3.1 \\
Spiral & 321 & 2.0 & 1.6 & 4.7 \\
roughing & 943 & 0.3 & 3.9 & 3.2 \\
\hline
\end{tabular}

Performance tests with or without zoom show that the CUDA kernel must be chosen depending on the simulation configuration. Conversely, it is not a problem with the CPU engine because the number of available threads is much lower than the number of positions. During the initial implementations, the acceleration factor was more important between GPU and CPU, but successive optimizations have, systematically, further improved the CPU engine rather than GPU implementations. The difference between the two versions is reduced over the optimizations. We thought that a fair and accurate comparison between CPU and GPU needs to have two optimized versions of the code for both architectures.

\subsection{2/64-Bit implementation}

Both configurations have been tested on the same case study in order to compare 32-bit and 64-bit performances. Achieving high performance on CUDA requires limiting access to the global memory, or in double precision, these accesses are doubled. In addition, the number of registers used in a kernel has a very important impact on the number of threads that can be launched by block, and therefore on performances. Moreover, only some CUDA cores can run 64-bit floating-point operations. The 64-bit implementation uses 59 registers against 33 for the 32-bit implementation, thus the potential parallelism is lower because the size of the register bank on each multiprocessor is limited.

As expected, the results (Tables 7 and 8) show that both GeForce and Quadro cards are less powerful in 64-bit computing. The loss of performance of 
the GeForce card in 64-bit computing is greater than the Quadro. Indeed, the Quadro is optimized for scientific calculations while the GeForce is a consumer card for video games. In terms of speed-up, results are of the same order with the theoretical speed-up established in section 6.1.

Table 7: 32/64-Bit speed-up with Coarse approach CAD/CAM configuration

\begin{tabular}{lccc}
\multicolumn{1}{c}{ Case } & Time (ms) & \multicolumn{2}{c}{ Quadro 4000 } \\
& CPU 64-bit & 32-bit & 64-bit \\
\hline Random & 366 & 8.5 & 5.3 \\
Spiral & 1061 & 6.4 & 4.3 \\
roughing & 7891 & 8.4 & 5.1 \\
Finishing & 11542 & 7.6 & 4.9 \\
\hline
\end{tabular}

Table 8: 32/64-Bit speed-up with Coarse approach GAMING configuration

\begin{tabular}{lccc}
\multicolumn{1}{c}{ Case } & Time (ms) & \multicolumn{2}{c}{ GeForce } \\
& CPU 64-Bit & 32-bit & 64-bit \\
\hline Random & 1260 & 28.3 & 12.8 \\
Spiral & 4634 & 47.8 & 9.1 \\
roughing & 25160 & 39.8 & 8.7 \\
Finishing & 30603 & 27.2 & 7.7 \\
\hline
\end{tabular}


In order to compare the devices with their best performances, speed-up relatives to the Xeon CPU computation time are proposed in Table 9. Calculations performed by the 32-bit GeForce card are about twice faster than 64-bit calculations of the Quadro. Theoretically, the speed-up with the 32-bit GeForce should be better and it could have been the case in a third configuration including both the Xeon CPU and the GeForce card. As discussed before, this raises the problem of computation accuracy but the improved algorithm (section 4) can ensure relatively low numerical disparities. One can imagine using 32-bit mode to explore different areas of the part. As the user is gradually zooming, the vertical amplitude of the simulation decreases, so the z-buffer lines height decreases as well as simulation error (section 4). Finally, a 64-bit computing simulation is launched once a specific area is chosen.

\begin{tabular}{lccc}
\multicolumn{2}{c}{ Table 9: } & 32/64-bit speed-up with Coarse approach \\
Case & Time (ms) & GeForce 560 Ti & Quadro 4000 \\
& CPU Xeon & 32-bit & 64 -bit \\
\hline Random & 366 & 7.6 & 5.3 \\
Spiral & 1061 & 10.1 & 4.3 \\
roughing & 7891 & 11.1 & 5.1 \\
Finishing & 11542 & 8.3 & 4.9 \\
\hline
\end{tabular}

\subsection{CAM software comparison}

On the other hand, an industrial configuration is proposed, which consists in the 3-axis machining of a plastic injection mold to produce polycarbonate optical lenses for ski mask. The finishing simulation is compared to an industrial CAM software simulation in terms of visible defects and computation time. The tool path contains 7.10e5 tool positions and the tool is considered as a canonical sphere.

Because of the numerical connection of CAD patches, a tangency discontinuity has been introduced in the toolpath along the vertical symmetry axis in the middle of the lens. The machining simulation should emphasize the resulting geometrical deviation. Despite using the best settings offered by the CAM 
software, the rendering of the simulation is not enough precise to detect the groove generated during tool path computation (Fig. 9). The processing time for this simulation is $450 \mathrm{~s}$. On the other hand, the proposed simulation allows without any zoom to highlight this defect in one run (Fig. 10) with a processing time of $44 \mathrm{~s}$.

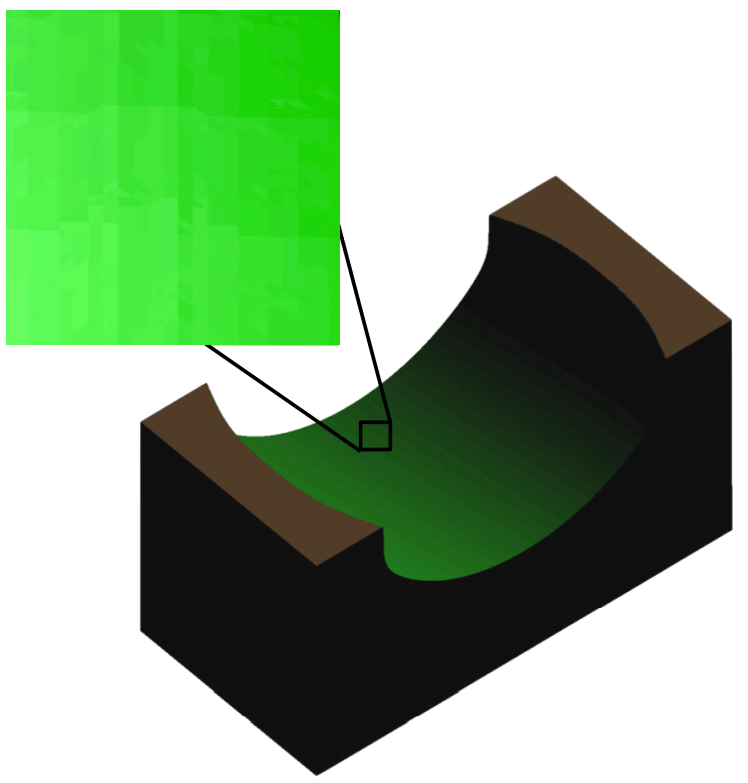

Figure 9: CAM software simulation

\section{Conclusion}

Machining simulations in CAM software are mainly used to detect collisions between the tool and the part on the whole part surface. It is difficult, if not impossible, to restrict the simulation to a delimited area in which the accuracy is much better to control the surface topography. To overcome this issue, this paper presents some opportunities to speed-up machining simulations and to provide multi scale simulations based on Nvidia CUDA architecture.

The results show that the use of the CUDA architecture can significantly improve performance on the computation time. However, if the granularity of 


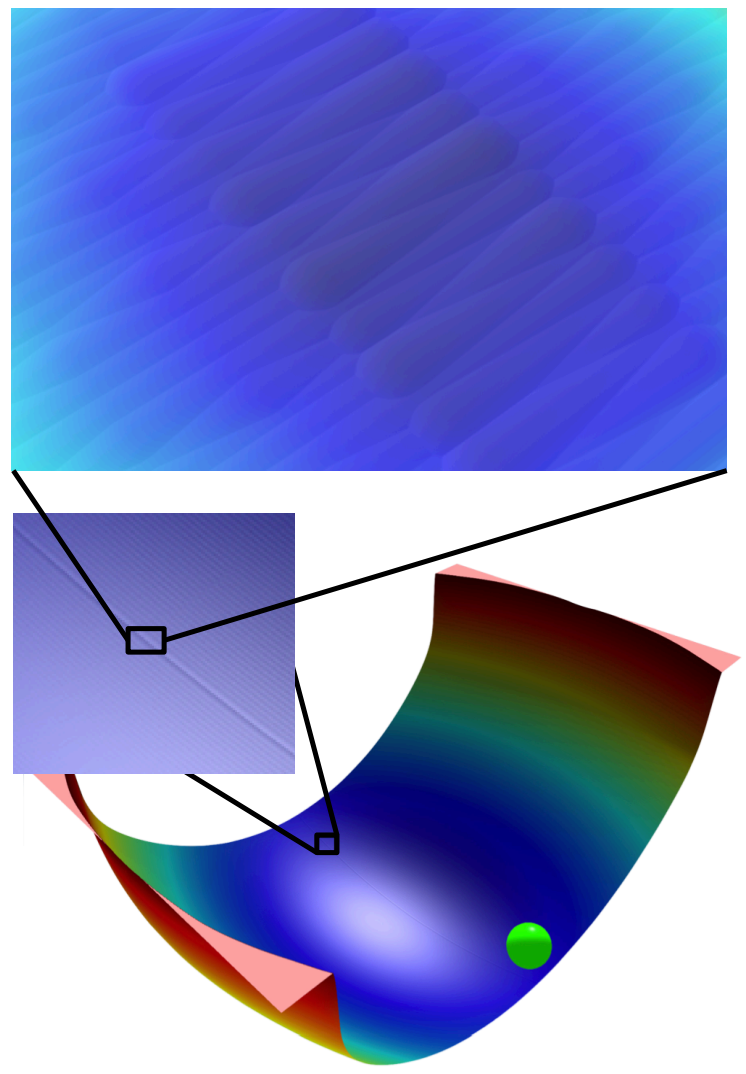

Figure 10: GPU mask simulation

tasks is not set correctly, the massively parallel CUDA architecture will not be used and the implementation may be slower than CPU. It is therefore necessary to adapt the parallelization strategy for the type of simulation, namely large scale or small scale.

Regarding the future works, the use of several 32-Bits GPUs in one hand and OptiX, the Nvidia ray tracing engine, in other hand, should improve the speedup. A faster simulation software could help to compute multi scale simulations with real time rendering or to improve the quality of the surface topography simulation by introducing stochastic behavior and simulate tool wear and the use of abrasive. 


\section{References}

[1] E. Budak, Analytical models for high performance milling Part I: cutting forces, structural deformations and tolerance integrity, International Journal of Machine Tools and Manufacture, 46 (12-13) (2006) 1478-1488

[2] E. Budak, Analytical models for high performance milling. Part II: process dynamics and stability, International Journal of Machine Tools and Manufacture, 46 (12-13) (2006) 1489-1499

[3] Y. Altintas, G. Stepan, D. Merdol, Z. Dombovari Chatter stability of milling in frequency and discrete time domain. CIRP Journal of Manufacturing Science and Technology, 1 (1) (2008) 35-44

[4] R. Jerard, R. Drysdale, K. Hauck, B. Schaudt, and J. Magewick Methods for detecting errors in numerically controlled machining of sculptured surfaces. IEEE Computer Graphics and Applications, 9 (1) (1989) 26-39

[5] D. Jang, K. Kim, and J. Jung. Voxel-based virtual multi-axis machining. International Journal of Advanced Manufacturing Technology, 16 (2000) 709-713

[6] Y. Quinsat, L. Sabourin, and C. Lartigue. Surface topography in ball end milling process: description of a 3D surface roughness parameter. Journal of Materials Processing Technology, 195 (1-3) (2008) 135-143

[7] W. He and H. Bin. Simulation model for CNC machining of sculptured surface allowing different levels of detail. The International Journal of Advanced Manufacturing Technology, 33 (2007) 1173-1179

[8] S. Lavernhe, Y. Quinsat, C. Lartigue, C. Brown. Realistic simulation of surface defects in 5-Axis milling using the measured geometry of the tool. International Journal of Advanced Manufacturing Technology, 74 (1-4)(2014) $393-401$ 
[9] Y. Quinsat, S. Lavernhe, C. Lartigue. Characterization of 3D surface topography in 5-Axis milling, Wear, 271(3-4) (2011) 590-595

[10] V. Morell-Gimenez, A. Jimeno-Morenilla, and J. Garcia-Rodrıguez. Efficient tool path computation using multi-core GPUs. Computers in Industry, 64 (1) (2013) 50-56

[11] Y. Takeuchi, M. Sakamoto, Y. Abe, R. Orita, and T. Sata. Development of a personal cad/cam system for mold manufacture based on solid modeling techniques. CIRP Annals - Manufacturing Technology, 38(1)(1898) 429-432

[12] H. Suzuki, Y. Kuroda, M. Sakamoto, S. Haramaki, and H. V. Brussel. Development of the CAD/CAM system based on parallel processing and inverse offset method. In Transputing'91 Proceeding of the world Transputer user Group (WOTUG) conference (1991)

[13] J. Kaneko and K. Horio. Fast cutter workpiece engagement estimation method for prediction of instantaneous cutting force in continuous multiaxis controlled machining. Procedia CIRP, 4(0)(2012) 151-156

[14] M. Inui, N. Umezu and Y. Shinozuka. A Comparison of two methods for geometric milling simulation accelerated by GPU. Transactions of the Institute of Systems, Control and Information Engineers, 26 (3) (2013) 95-102

[15] CUDA C Programming Guide. http://developer.nvidia.com/cuda/ cudadownloads

[16] R. Farber, R. CUDA Application Design and Development. Elsevier Science, 2011

[17] I. Wald, S. Boulos, and P. Shirley. Ray tracing deformable scenes using dynamic bounding volume hierarchies, ACM Transactions on Graphics, 26(1)(2007)

[18] Salembier and L. Garrido. Binary partition tree as an efficient representation for image processing, segmentation, and information retrieval. IEEE Transactions on Image Processing, 9(4)(2000), pp. 561-576 
[19] N. Whitehead and A. Fit-Florea. Precision and Performance: Floating Point and IEEE 754 Compliance for NVIDIA GPUs, nVidia technical white paper, 2011 